Comment les sociétés « se débarrassent de leurs vivants » : dangerosité et psychiatrie, la donne contemporaine

How Sociery gets rid of the living : dangerosity and psychiaty in our

contemporary world

\title{
Véronique Voruz
}

\section{(2) OpenEdition \\ Journals}

\section{Édition électronique}

URL : http://journals.openedition.org/conflits/18912

DOI : 10.4000/conflits. 18912

ISSN : $1777-5345$

Éditeur:

CCLS - Centre d'études sur les conflits lilberté et sécurité, L'Harmattan

\section{Édition imprimée}

Date de publication : 31 décembre 2014

Pagination : 203-223

ISBN : 978-2-343-05760-6

ISSN : 1157-996X

\section{Référence électronique}

Véronique Voruz, «Comment les sociétés « se débarrassent de leurs vivants » : dangerosité et psychiatrie, la donne contemporaine », Cultures \& Conflits [En ligne], 94-95-96 | été-automne-hiver 2014, mis en ligne le 20 février 2016, consulté le 30 mars 2021. URL : http://journals.openedition.org/ conflits/18912 ; DOI : https://doi.org/10.4000/conflits.18912 


\section{Comment les sociétés}

\section{« se débarrassent de leurs vivants »: dangerosité et psychiatrie, la donne contemporaine 1}

\section{Véronique VORUZ}

Véronique Voruz est mầtre de conférences en droit et criminologie à l'École de Droit de l'Université de Leicester au Royaume-Uni.

Il y a deux institutions préposées aux dangers représentés par les individus : médecine et droit. Le psychiatre est le préposé aux dangers individuels. ${ }^{2}$

T a gestion des risques est au principe des gouvernementalités biopolitiques - telle est la grille d'intelligibilité qu'utilisent de nombreux penseurs inspirés par Michel Foucault ${ }^{3}$ pour « diagnostiquer » le présent ${ }^{4}$ depuis plus de deux décennies. Ces dernières années, plusieurs auteurs se sont cependant attachés à démontrer que si les logiques gouvernementales restaient articulées autour des concepts de risque, d'assurance et de prévention pour gouverner les populations, un changement de tonalité s'était néanmoins produit : nous serions passés de la gestion des risques à une «version de précaution qui, en un sens, absolutise la menace, la rend absolument anormale et intolérable [...]

1. Une première version de ce texte fut présentée au service de psychiatrie des Hôpitaux Universitaires de Genève le 17 avril 2013 sous le titre «La dangerosité en psychiatrie ».

2. Foucault M., «Table Ronde sur l'expertise psychiatrique » in Foucault M., Dits et écrits t. 2, Paris, Gallimard, 1994, p. 665.

3. C'est la thèse défendue par François Ewald à partir du travail de Foucault, notamment dans Ewald F., "Norms, Discipline, and the Law ", Representations, n 30, 1990, pp. 138-161. Pour ce qui est de la criminologie, voir O’Malley P., Risk, Uncertainty and Government, London, GlassHouse Press, 2004, ouvrage assez représentatif de la criminologie dite critique dans le monde anglophone, dont les autres tenants les plus connus sont Nikolas Rose et David Garland. Voir aussi plus généralement Burchell G., Gordon C. et Miller P. (eds.), The Foucault Effect, Harvester, 1991, point de départ des governmentality studies.

4. Pour une exposition de ce qu'est un diagnostic du présent, voir Deleuze. G., «Qu'est-ce qu'un dispositif », in Michel Foncault philosophe, Paris, Seuil, 1989. 
la précaution tient [le risque] pour une monstruosité qu'il faut annuler ${ }^{5}$ ». Ce serait dans cette perspective de précaution que les psychiatres seraient de plus en plus convoqués par les politiques gouvernementales, d'une part au dépistage d'une dangerosité individuelle qui pourrait faire d'un fou un criminel 6, mais d'autre part, et bien plus fondamentalement, à la création, à l'entretien et à la légitimation d'un continuum illimité dans le temps aussi bien que dans l'espace (suivis socio-judiciaires et peines de sûreté) permettant le contrôle continu de groupes d'individus dits à risque au nom du soin ${ }^{7}$.

La dangerosité, signifiant opératoire de nos pratiques pénales, inclut aujourd'hui aussi bien les passages à l'acte violents que le récidivisme ou encore les comportements indésirables. Foucault l'a démontré dans ses généalogies, c'est à partir du « grand événement criminel, extrêmement violent et extrêmement rare ${ }^{8}$ » que la classe délinquante a été constituée comme dangereuse dans son intégralité. Or, comme il s'est employé à le démontrer dans plusieurs textes et interventions, qui datent des années 1970 mais qui restent d'une actualité brûlante, la dangerosité est une notion qui n'est ni juridique ni psychiatrique 9 . Sa fonction première est, à partir de quelques cas "monstrueux » et exceptionnels mis en exergue par la littérature médico-légale 10 et les discours politico-médiatiques, de légitimer la «manière dont [les sociétés] se débarrassent non pas de leurs morts, mais de leurs vivants... ${ }^{11}$ ». Cet article se propose de faire retour sur les positions sans compromis du philosophe pour en retrouver le tranchant aujourd'hui émoussé 12 par d'innombrables lectures des pratiques pénales en termes managériaux et actuariels ${ }^{13}$.

5. Doron C.-O, «Une volonté infinie de sécurité : vers une rupture générale dans les politiques pénales ? ", in Chevallier P. et Greacen T. (eds.), Folie et justice : relire Foucault, Toulouse, Éditions Érès, 2009, p. 180.

6. Sur les rapports entre justice et psychiatrie, voir l'excellente collection de textes recueillis dans Folie et justice, op. cit.

7. Pour le passage de l'individu dangereux à l'individu à risques, voir Castel R., «De la dangerosité au risque ", Actes de la recherche en sciences sociales, vol. 47, n 1, 1983.

8. Foucault M. "L'évolution de la notion d'“individu dangereux" dans la psychiatrie légale du XIXe siècle » in Foucault M., Dits et écrits t. 3, Paris, Gallimard/Le Seuil, p. 446.

9. Dans «Enfermement, psychiatrie, prison », après avoir posé que la vocation de la psychiatrie était l'hygiène publique et l'ordre social (in Foucault M., Dits et écrits t. 3, op. cit., p. 334), Foucault ajoute : « être dangereux, ce n'est pas un délit. Être dangereux, ce n'est pas une maladie. Ce n'est pas un symptôme. Eh bien, on arrive, comme à une évidence, et cela depuis plus d'un siècle, à faire fonctionner la notion de danger, par un renvoi perpétuel du pénal au médical et réciproquement. Le pénal dit : écoutez, celui-là, je ne sais pas très bien quoi en faire, je voudrais avoir votre avis - est-ce qu'il est dangereux ? Et le psychiatre, si on lui dit : mais enfin vous allez répondre à cette question ? va répliquer : évidemment. Le “danger”, ce n’est pas une notion psychiatrique - mais c'est la question que me pose le juge. Et hop ! Si l'on considère l'ensemble, on s'aperçoit que tout cela fonctionne à la notion de danger. », p. 343.

10. Voir par exemple Archambaut J-C., L'expertise psychiatrique face à la dangerosité et à la récidive des criminels, Paris, Odile Jacob, 2012 et Zagury D. et Assouline F., L'énigme des tueurs en série, Paris, Plon, 2008. On peut aussi consulter le site de Stéphane Bourgoin, Le troisième cill, dédié aux tueurs en série.

11. "Normalisation et contrôle social », in Foucault M., Dits et écrits t. 2, Paris, Gallimard/Le Seuil, 1994, p. 319.

12. Voir sur ce point la position de Jacques Donzelot dans son entretien avec Colin Gordon, Donzelot J. et Gordon C., « Governing Liberal Societies : The Foucault Effect in the English- 
Reprenons donc l'analyse à son point de départ. Juridiquement, l'architecture du code pénal français repose sur la notion de responsabilité. Le " constat de l'irresponsabilité est déterminé par l'existence ou non d'un trouble mental, et non par l'évaluation d'une quelconque dangerosité, qu'elle soit psychiatrique ou criminologique ${ }^{14} »$. La dangerosité n'est pas non plus une condition médicale qui serait repérable à partir de certains symptômes précis. Il s'agit d'une notion criminologique, d'un instrument de politique criminelle indexée aux probabilités de passage à l'acte ou de récidive. Son champ est ouvertement présenté comme celui de la gestion des risque : le risque représenté par les délinquants pour la dangerosité criminologique, par les personnes souffrant de troubles mentaux pour la dangerosité psychiatrique ${ }^{15}-$ si tant est qu'il subsiste une différence entre ces deux catégories. En dépit du caractère hybride, mal défini de cette notion, la mission des psychiatres est double dans le contexte pénal. D’une part, ils sont appelés à déterminer la responsabilité ou l'irresponsabilité d'un infracteur par référence au discernement en application de l'article 122 du code pénal ; d'autre part la demande d'expertise formulée par le juge contient traditionnellement une série de questions ayant trait à « l'état dangereux » de l'intéressé 16 .

L'intervention de la psychiatrie dans le domaine du droit, aujourd'hui une «objectivité » incontestée de nos pratiques, est pourtant relativement nouvelle : elle date du XIXe siècle ${ }^{17}$. Cette intervention produit un redoublement dans l'appréhension de l'infracteur: responsabilité et imputabilité pour ce qui est de l'acte criminel, risque et dangerosité pour ce qui est de la subjectivité dite criminelle. Ce redoublement d'une qualification de l'acte par une interprétation de la subjectivité censée y être corrélée a pu être qualifiée par Foucault «d'autre lecture 18 ».

Speaking World ", Foncault Studies 5, 2008, pp. 48-62, ou encore Hutchinson S., "Countering Catastrophic Criminology : Reform, Punishment and the Modern Liberal Compromise ", Punishment and Society, 8(4), 2006, pp. 443-67.

13. Voir par exemple Simon J., Governing through Crime, New York, Oxford University Press, 2007.

14. Rapport de la Haute Autorité de Santé «Audition publique dangerosité et troubles mentaux : Dangerosité psychiatrique : étude et évaluation des facteurs de risque de violence hétéroagressive chez les personnes ayant des troubles schizophréniques ou des troubles de l'humeur », 2011, http://www.has-sante.fr/portail/upload/docs/application/pdf/2011-07/evaluation_de_la_dangerosite_psychiatrique_-_rapport_dorientation.pdf (consulté le 15 avril 2013), p. 7, ci-après HAS 2011.

15. Sur les rapports entre psychiatrie et gestion des risques, voir Castel R., La gestion des risques, Paris, Éditions de Minuit, 1981.

16. Pour une liste des questions traditionnellement posées dans le cadre des expertises, voir le Rapport Commission Santé-Justice de 2005, «Santé, justice et dangerosités : pour une meilleure prévention le récidive ", http://www.sante.gouv.fr/htm/actu/sante_justice/rapport.pdf (consulté le 15 avril 2013), pp. 29-30, ci-après Rapport Santé-Justice.

17. Pour une histoire des rapports entre justice et psychiatrie, voir Renneville M., Crime et folie : deux siècles d'enquêtes médicales et judiciaires, Paris, Arthème Fayard, 2003.

18. «...au début du XIXe siècle, toutes les pratiques policières de sélection sociale [mendiants, oisifs, vagabonds] ont été réintégrées à l'intérieur de la pratique judiciaire parce que, dans l'État napoléonien, police, justice et institutions pénitentiaires ont été articulées les unes sur 
Plus généralement, la dangerosité est l'instrument conceptuel au moyen duquel le lien entre droit et psychiatrie, deux disciplines autrefois distinctes, s'est noué ; il a de même permis la naissance de la criminologie comme «science » de la dangerosité ainsi que la constitution de la forme de subjectivité attenante, la subjectivité dite criminelle. C'est depuis que l'on a confié au pouvoir judiciaire - en Europe généralement au XIXe siècle - la mission non plus seulement de juger les infracteurs mais aussi d'aménager et de gérer la peine selon le principe de l'individualisation des sentences que cette notion de politique criminelle a pu se développer, jusqu'à en devenir centrale pour tout ce qui est des décisions pré- et post-sentencielles ${ }^{19}$. Foucault a fait une généalogie de ce «doublage juridico-clinique 20 » dans son intervention de Toronto en 1977, intitulée "L'évolution de la notion d' "individu dangereux" dans la psychiatrie légale au XIXe siècle $21 »$. Je commenterai ce texte ainsi que quelques autres dans le troisième temps de cet article.

Dans un premier temps, je voudrais faire un bref survol des différentes facettes de la dangerosité telle qu'elle se présente dans les discours et pratiques actuels. Le deuxième temps sera consacré aux stratégies réformistes qui s'y opposent. Mes illustrations sont tirées essentiellement des situations française et anglaise, sachant qu'il existe une assez grande continuité entre les politiques criminelles des différents pays européens.

\section{Droit pénal et dangerosité}

\section{Le contexte contemporain}

Les systèmes pénaux des démocraties de la modernité tardive 22 se sont inscrits les uns après les autres dans une logique de prévention indexée sur la dangerosité avérée ou supposée des délinquants. Les législations européennes ont par exemple pour point commun d'indexer la durée et le régime des peines carcérales de moins en moins sur le principe d'une proportionnalité entre sévérité de l'acte criminel et sanction et de plus en plus sur la dangerosité, réelle ou perçue, de l'infracteur, donc en « incriminant la probabilité crimi-

les autres et, au moment même où ces pratiques s'intégraient à la pratique judiciaire, donc policière, au même moment où sont apparues, pour les justifier, pour les doubler, pour en donner une autre lecture (pas pour leur donner une autre lisibilité) des catégories psychologiques, psychiatriques, sociologiques nouvelles. » in Foucault M., " Normalisation et contrôle social », op. cit., p. 317.

19. Voir sur ce point notamment Généalogie de la défense sociale en Belgique (1880-1914): Textes recueillis par Françoise Tulkens, Bruxelles, E. Story-Scientia, 1988. Il s'agit des travaux du séminaire qui s'est tenu à Louvain en 1981 sous la direction de Michel Foucault.

20. Expression employée par Foucault dans « Normalisation et contrôle social », op. cit., p. 316: "les catégories juridiques de l'exclusion ont régulièrement leurs corrélatifs médicaux ou cliniques. »,.

21. Foucault M., «L'évolution de la notion d'“individu dangereux" dans la psychiatrie légale du XIXe siècle » in Foucault M., Dits et écrits t. 3, op.cit., pp. 443-464.

22. Pour une caractérisation de ces systèmes voir Garland D., The Culture of Control, Oxford, Oxford University Press, 2001. 
nelle 23 ». La jurisprudence de la Cour Européenne des Droits de l'Homme elle-même s'attache, depuis quelques années, à redéfinir le principe de proportionnalité entre peine et crime en termes d'une proportionnalité entre peine et risque (de récidive, etc.) ${ }^{24}$. C'est donc la subjectivité criminelle plus que l'acte qui oriente nos pénologies.

En Angleterre, les Dangerous Offender Policies se multiplient depuis l'introduction dans le Criminal Justice Act [1991] de la possibilité d'imposer une peine carcérale qui fasse échec au principe de proportionnalité entre l'infraction et la peine, principe que cette même loi posait par ailleurs, lorsque cela est justifié pour la protection du public et à la discrétion du juge ${ }^{25}$. Cette modalité d'établissement de la durée de la peine est passée du statut d'exception à celui de norme avec le Criminal Justice Act [2003], qui introduit et consolide toute une série de peines prolongées, à durée indéterminée ou encore à vie (à savoir sans aucune possibilité de libération conditionnelle) pour les criminels dits à risque. Dans le cas de ces peines dites IPPs [Imprisonment for Public Protection], il incombe à l'infracteur de démontrer qu'il n'est plus dangereux avant d'être remis en liberté après avoir servi sa peine, et ce quelle que soit la durée de la peine initiale. Ce sont à des expertspsychiatres qu'il appartient d'évaluer cette dangerosité. Des milliers d'infracteurs ${ }^{26}$ sont aujourd'hui détenus dans les prisons anglaises pour une durée indéterminée après qu'ils ont servi leurs peines car ils ne peuvent pas prouver qu'ils ne sont plus dangereux, n'ayant pas accès aux techniques de réhabilitation qui sont uniquement ouvertes aux prisonniers dont la durée de la peine est définie d'emblée comme longue. L'évidente injustice de cette situation comment rapporter la preuve de son inoffensivité sans les certificats délivrés par les institutions pénitentiaires - ainsi que les coûts exorbitants entraînés par ces mesures populistes ont fini par donner lieu au Legal Aid, Sentencing and Punishment of Offenders Act, promulgué le $1^{\mathrm{er}}$ mai 2012, qui a aboli les IPPs pour les infracteurs condamnés après le 1er décembre 2012. C’est comme toujours le souci budgétaire qui parvient à entraîner l'assouplissement des mesures les plus liberticides ${ }^{27}$.

23. L’expression est de Denis Salas, «La vérité, la sûreté, la dangerosité. Trois généalogies à l'épreuve des débats contemporains ", in Chevallier P. et Greacen T. (eds.), Folie et justice..., op. cit., p. 213.

24. Voir Player E. "Remanding Women in Custody: Concerns for Human Rights », Modern Law Review, 70(3), 2007, pp. 402-426.

25. Pour une analyse fine et précise des récents développements du processus de criminalisation en Angleterre, voir Lacey N., "Space, time and function: intersecting principles of responsibility across the terrain of criminal justice ", Crim Law and Philos, vol. 1, 2007, pp. 233-250.

26. En septembre 2013 il y avait 13000 prisonniers détenus sous le régime de peines à durée indéterminée pour la protection du public (House of Commons Standard Note SN/HA/6086).

27. Cela avait déjà été le cas en 1990 quand le gouvernement conservateur avait conclu à la nécessité de décarcérer pour faire des économies, ainsi que le montre le très explicite titre du projet de loi précédant le CJA de 1991: «Imprisonment: an expensive way of making bad people worse ». 
On y note aussi une convergence de plus en plus nette entre le traitement des criminels et celui des personnes souffrant de «troubles » de la santé mentale 28 , avec par exemple la possibilité de détenir de façon préventive les personnes souffrant d'un trouble du comportement considéré come dangereux (par exemple, le Dangerous and Severe Personality Disorder). Toujours en Angleterre, la réforme de 2007 du Mental Health Act [1983] a introduit les injonctions de soins dans la communauté [Community Treatment Orders]. Ces nouveaux pouvoirs s'appliquent en matière pénale comme civile et se combinent avec le pouvoir de ré-hospitaliser le patient et de le contraindre à suivre un traitement médical lorsque ce traitement n'est pas observé dans la communauté. De même, l'hospitalisation d'office n'est plus soumise à la condition que le patient soit traitable, du moment qu'il y a traitement approprié [Appropriate Treatment Test].

Quant à la France, longtemps à la traîne en ce domaine par rapport aux pays anglo-saxons, la loi Dati sur la rétention et la surveillance de sûreté [2008] introduit des mesures indexées sur la dangerosité supposée du criminel, c'est-à-dire, selon la définition qu'en donne la Commission nationale consultative des droits de l'homme, sur « la prédiction aléatoire d'un comportement futur 29 », et non pas sur l'acte. Pour ce qui est de la place de la psychiatrie dans son rapport au droit pénal, le rapport de la Commission SantéJustice, intitulé «Santé, justice et dangerosité : pour une meilleure prévention de la récidive » (2005) a été suivi d'un rapport de la Fédération Française de Psychiatrie en 2007 sur l'expertise psychiatrique pénale ${ }^{30}$. L'objectif de ces développements était d'affiner l'évaluation des dangerosités ${ }^{31}$ psychiatrique et criminologique des infracteurs. Les angoisses suscitées par une série de faits divers à sensation ont donné l'impulsion nécessaire à la loi du 5 juillet 2011, qui autorise les soins psychiatriques sans consentement sous contrôle du préfet dans le cas d'infractions pénales ${ }^{32}$. Ces derniers textes, pris avec la loi de 2007 sur les peines planchers ${ }^{33}$, indiquent clairement que la justice pénale

28. Voir par exemple le débat sur l'internement préventif des personnes diagnostiquées comme souffrant de Dangerous and Severe Personality Disorder, débat qui fait rage en Angleterre depuis 1999, Seddon T., "Dangerous Liaisons", Punishment E Society, vol. 10-3, 2008, pp. 301-317.

29. Cité dans la Conférence de Consensus pour une nouvelle politique publique de prévention de la récidive, Rapport présenté au Premier Ministre Jean-Marc Ayrault le 20 février 2013, http://conference-consensus.justice.gouv.fr (consulté le 15 juillet 2014), p. 29.

30. Rapport de la Fédération Française de Psychiatrie sur l'expertise psychiatrique pénale [2007] http://www.has-sante.fr/portail/upload/docs/application/pdf/epp-rapport_de_la_commission-version_finale_pour_mel.pdf (consulté le 12 août 2013).

31. «Ainsi, l'éventuelle dangerosité d'un individu auteur d'une infraction doit être prise en considération, à la fois lors de l'examen de la déclaration d'irresponsabilité pénale pour troubles mentaux et de ses suites, au moment du choix, de l'exécution et de l'aménagement de la peine et lors d'un suivi postérieur à l'exécution de cette dernière. » (Rapport Santé-Justice, op. cit., p. 38).

32. L'article 3 de la loi pose la possibilité d'admission en soins psychiatriques sur décision du représentant de l'Etat et sans le consentement de l'intéressé. Il s'agit dans ce cas d'une intervention du préfet au titre de la sûreté des personnes et de la protection de l'ordre public.

33. Les peines planchers ont été supprimées à compter du 1er octobre 2014. 
française a rattrapé son « retard » en matière de contrôle social ${ }^{34}$. On a ainsi assisté, dans les termes du rapport sur la récidive rendu au Premier Ministre du moment, Jean-Marc Ayrault, en février 2013, à la transition « d'une justice de responsabilité à une justice de sûreté 35 ».

\section{Droit pénal et psychiatrie}

Dans ce contexte d'une « justice de sûreté », c'est la subjectivité criminelle qui est au centre des dispositifs de sécurité. Les psychiatres sont donc en première ligne pour ce qui est, ostensiblement, d'évaluer la dangerosité des personnes souffrant de troubles mentaux, et avec moins d'éclat mais plus d'impact, de « légitimer la peine-traitement et le continuum peine-mesure de sûreté 36 ».

Il ne s'agira pas ici de prendre parti pour ou contre le fait que la psychiatrie soit en position d'avoir à répondre de la dangerosité psychiatrique et/ou criminologique des personnes sous main de justice, ou même des patients rencontrés dans le cadre d'un travail purement clinique (et non dans un contexte médico-légal). C'est un débat stérile; il est évident que si le débat est cantonné à la question du pour ou contre la dangerosité, le bon sens, cet imparable éteignoir du désir de savoir, ne peut que se prononcer en faveur du dépistage des individus dangereux ainsi que de leur mise hors d'état de nuire.

Il me parait plus opportun d'épingler ici la réorganisation de la psychiatrie autour de ces impératifs d'évaluation aux dépens de la clinique, sanctions judiciaires à l'appui 37 . Vu la crise épistémologique 38 qu'elle traverse

34. Voir, pour une analyse critique des développements sécuritaires de la justice pénale française, Salas D., La volonté de punir : essai sur le populisme pénal, Paris, Arthème Fayard/Pluriel, 2010, et La justice dévoyée : critique des utopies sécuritaires, Paris, Éditions des Arènes, 2012.

35. Conférence sur la récidive, op. cit., p. 28.

36. Danet J., "Droits de la défense et savoirs sur le crime ", in Justice et folie, op. cit., p. 85.

37. Ainsi qu'en témoigne la condamnation à une peine d'un an d'emprisonnement avec sursis pour homicide involontaire prononcée le 18 décembre 2012 par le tribunal correctionnel de Marseille à l'encontre de la psychiatre Danièle Canarelli suite à l'assassinat commis par un de ses patients. La condamnation a été prononcée suite à un rapport d'expertise très sévère pour la psychiatre de soins et sur la base de la loi Fauchon [2000]. Cette dernière requiert une faute caractérisée par une «violation manifestement délibérée d'une obligation particulière de prudence ou de sécurité » pour engager la responsabilité des décideurs publics au titre des conséquences non intentionnelles de leurs décisions. L'expert a argumenté que le $\mathrm{D}_{\mathrm{r}}$ Canarelli avait commis une faute caractérisée dans le suivi de son patient Joël Gaillard de par son « laxisme » dans le traitement d'un individu dangereux, concluant à une discordance entre le suivi thérapeutique et la gravité du cas. Il s'agit donc ici de la cristallisation judiciaire d'un conflit entre les psychiatres de soin, qui font prévaloir la dimension clinique, et les experts psychiatres et le pouvoir judiciaire, qui cèdent à la pression des discours sécuritaires. Or ces derniers refusent qu'il n’y ait pas de coupable lorsque le 'risque zéro' n'a pas été obtenu par l'action combinée de la justice et de la médecine. Un nouveau volet s'ouvre ainsi dans les rapports entre la psychiatrie et le droit. Il est à noter cependant que la psychiatre a reçu le soutien des principaux syndicats de psychiatres : "Nous ne sommes pas des policiers. Entre le patient et le psychiatre, tout doit être basé sur la confiance et le soin thérapeutique, pas sur la sécurité », déclare à cette occasion Olivier Labouret, Président de l'Union syndicale de la psychiatrie, au journal 
aujourd'hui, crise dont les effets viennent s'ajouter « à la défaite d'une psychiatrie du traitement au profit d'une psychiatrie de sûreté 39 », la dimension clinique de la psychiatrie se voit reléguée bien derrière la demande hygiéniste faite aux psychiatres d'avoir à " participer à un projet de contrôle des comportements 40 ». Foucault disait déjà en 1976 que nous avions « une justice qui s'innocente de punir en prétendant traiter le criminel 41 »; l'affaiblissement théorique actuel du discours clinique, dont les concepts de plus en plus «scientifiques » font de moins en moins place aux possibilités de subjectivation des personnes rencontrées, renforce encore le caractère objectivant des politiques pénales. Pour beaucoup de psychiatres, une telle approche, loin d'être préventive, ne peut qu'accroître l'éventualité d'un passage à l'acte et en tout cas entraîne ségrégation et déprise du lien social. Que ce soit par l'anamnèse, la construction d'un lien transférentiel stabilisant ou l'élaboration en paroles d'une compulsion à agir, le travail thérapeutique de longue durée est ce qui permet une réelle prévention de la récidive, car il ouvre aux infracteurs l'accès à de nouvelles lignes de subjectivation. Par contraste, les évaluations psychiatriques ou psychologiques se résument pour les sujets concernés en un impératif d'avoir à obéir aux prescriptions comportementales des acteurs du monde socio-judiciaire ${ }^{42}$, injonction dont l'effet surmoïque est évident.

Comment, donc, aborder la dangerosité, pierre d'angle du champ pénal, et dont la conséquence logique est la déshumanisation des infracteurs ? Cette question se décline en deux dimensions :

Comment interpréter la fascination pour la dangerosité, la monstruosité supposée des malades mentaux, fascination qui dans les médias informationnels comme culturels se substitue au désir de savoir ? Nos sociétés nous exposent à de bien plus sérieux dangers, statistiquement parlant, que ceux posés par les dits malades mentaux.

Le Monde du 18 décembre 2012, http://www.lemonde.fr/societe/article/2012/12/18/la-psychiatrie-n-est-pas-une-science-exacte_1790238_3224.html. De plus, la Cour d'appel d'Aixen-Provence a prononcé, le lundi 31 mars 2014, la prescription de l'action publique dans ce dossier.

38. Comme l'indique la multitude de commentaires, américains comme européens, émanant de praticiens comme d'usagers, prédisant l'échec du DSM-5 ainsi que la disparition du paradigme psychiatrique en vigueur au profit des neurosciences, paradigme soutenu par le National Institute of Mental Health américain en la personne de son directeur, Thomas Insel, et subventionné par le projet BRAIN du gouvernment Obama. Voir sur ce point la position de principe d'un certain courant de la psychiatrie anglaise: Bracken P. et al, « Beyond the Current Paradigm », The British Journal of Psychiatry, 201, 2012, pp. 430-434.

39. Salas D., op. cit., p. 207.

40. Ibid., p. 212.

41. Foucault M. "L’angoisse de juger » (entretien avec R. Badinter et J. Laplanche) in Foucault M., Dits et écrits t. 3, op. cit., p. 290.

42. Voir sur ces points Biagi-Chai F., Le cas Landru à la lumière de la psychanalyse, Paris, Imago, 2007. À partir de plusieurs cas célèbres, la psychiatre y démontre que la prévention du récidivisme dépend avant tout d'un engagement au cas par cas avec la subjectivité criminelle prise dans sa logique structurale. 
Que peut faire la psychiatrie, discipline divisée contre elle-même (entre experts et cliniciens) par la demande qui lui est adressée d'avoir à se prononcer sur le degré de risque qu'un patient ou délinquant représente pour la société tout en devant assurer les soins ? Et la profession psychiatrique saurat-elle éviter une dérive de la psychiatrie légale à l'américaine, qui a sécurisé sa pratique en substituant les outils actuariels au jugement clinique, mettant ainsi les praticiens à l'abri d'avoir à répondre judiciairement de potentielles erreurs de jugement?

\section{Fascination}

Pour ce qui est de la fascination, le texte produit par Archambaut, un expert psychiatre français, fort de 20 ans de service médico-légal, L'expertise psychiatrique face à la dangerosité et à la récidive des criminels, est très éclairant ${ }^{43}$. Le Dr Archambaut est expert-psychiatre près de la Cour d'appel de Paris, agréé par la Cour de cassation ainsi que la Cour Pénale Internationale, il est aussi Vice-Président du Conseil National des compagnies d'experts de justice (CNCEJ). Or son livre est une liste des crimes les plus horribles qui soient, décrits dans leurs détails les plus graphiques par l'auteur, non sans une certaine délectation. La table des matières ressemble à un best-of des films d'horreur hollywoodiens : «femmes perverses », « infanticides », « viols collectifs », " meurtre et cannibalisme », même Guantanamo y trouve sa place. L'analyse clinique, si tant est qu'il y en ait une, est d'une pauvreté atterrante. Le texte offre en pâture au lecteur immanquablement fasciné les fruits du regard soi-disant objectif de l'expert sur ces nouveaux « damnés de la terre ». Suggérée à chaque ligne est l'idée que, face à ces désordres narcissiques, pervers, irraisonnés, ces jouissances tous azimuts, l'expert « chevronné » est central au fonctionnement de la justice ; sur lui repose l'objectivité médicale ainsi que la prévention du pire. On peut aussi citer ici la position de Daniel Zagury, autre expert au centre de l'actualité criminologique, même si elle est plus mesurée. En effet Zagury a soin de mettre en garde contre l'instrumentalisation de la psychiatrie, à qui il est demandé de dédifférencier tout trouble de la personnalité ou de la sexualité des maladies mentales pour glisser « de l'enfermement de sûreté au soin à perpétuité 44 ». D’autre part il se targue « de débusquer la fascination », de révéler par son analyse le « fonctionnement psychique en perdition » là où le commun des mortels ne voyait que « la machinerie diabolique d'une intelligence du mal 45 ». Cette fascination reste cependant très présente dans les titres choisis : les mots "vampire », " génie du mal », " diabolique », " horreur », reviennent fréquemment sous sa plume, et les analyses cliniques n'apportent que peu d'éléments faisant signe d'une autre causalité que celle d'une jouissance malsaine.

43. Archambaut J-C., op. cit.

44. Voir Zagury D., «Pratiques et risques de l'expertise psychiatrique », in Justice et folie, op. cit., p. 96.

45. Zagury D. et Assouline F., op. cit., p. 9. 
C'est cette fascination pour la monstruosité supposée des fous criminels, pervers et terroristes, qui donne consistance aux discours sur la dangerosité et légitime les politiques privatives de droits. Elle s'accompagne de séries de revendications pour que soient mises en place les politiques nécessaires à l'avènement d'une société dont « risque zéro » serait le nom, voile utilitariste de la haine déchaînée par l'altérité desdits criminels.

\section{Que peut faire la psychiatrie : Réformisme et «politique de la vérité »}

Foucault l'a développé dans ses cours et interventions qui ont fait suite à son élaboration du pouvoir disciplinaire : les pratiques pénales actuelles prennent la forme de dispositifs de sécurité ${ }^{46}$, de dispositifs de gouvernement des risques dont font partie les technologies de gestion des individus appartenant à la catégorie dite des individus dangereux. Face à de tels dispositifs, au moins deux types de stratégies de résistance sont possibles :

- Le réformisme, qui consiste à opérer dans le cadre des « objectivités » qui définissent les termes du débat, et ce afin de limiter les excès attentatoires aux libertés individuelles ;

- Mais aussi ce que Foucault a pu appeler la politique de la vérité ${ }^{47}$, qui vise à fragiliser une vérité hégémonique, en apparence objective et incontestable. Dans une telle perspective de problématisation des objectivités plutôt que de réformisme, le but est de rendre visibles les lignes de fuite qui organisent les dispositifs de sécurité afin de réveiller les acteurs du théâtre médico-légal à leurs responsabilités éthiques.

Les deux stratégies ne sont pas exclusives, on peut être réformiste au jour le jour, mais sans perdre de vue la politique de la vérité. Voici par exemple comment, dans son ouvrage sur les prisons, Boullant formule la position foucaldienne sur ce point : «Des réformes peuvent et doivent être entreprises, mais dans le cadre plus radical d'une refondation de l'ensemble du système pénal, lequel est à changer de fond en comble. [...] Il n'y a pas pour [Foucault] de contradiction entre la radicalité d'une approche globale et la ponctualité et l'urgence des réformes 48 ».

46. Voir Foucault M., Sécurité, Territoire, Population, Paris, Gallimard/Seuil, 2004.

47. Foucault emploie cette expression dans sa conférence de 1978 «Qu'est-ce que la critique ? Critique et Aufklärung ", Bulletin de la société française de philosophie, 84e année, n², AvrilJuin 1990, p. 39. Pour une élaboration théorique du rapport entre réformisme et politique de la vérité, voir Voruz V., "Politics in Foucault's later Work : A Philosophy of Truth; or Reformism in Question ", Theoretical Criminology, vol. 15-1, 2011, pp. 47-65.

48. Boullant F., Michel Foucault et les prisons, Paris, Presses Universitaires de France, 2003, p. 110. 
$\mathrm{Au}$ titre du réformisme, nous pouvons constater de légères avancées en Angleterre comme en France. En Angleterre, des psychiatres proches du Critical Psychiatry Network s'opposent sans relâche à la médicalisation de la souffrance psychique et répondent à toutes les consultations gouvernementales sur l'uniformisation des best practices. Ils viennent ainsi de finir la première évaluation des injonctions de soin et contestent leur efficacité thérapeutique - statistiques à l'appui ${ }^{49}$. Cette étude a été reprise par le groupe de travail sur le Mental Health Act [2007] de la Chambre des Communes ${ }^{50}$, qui recommande donc dans son rapport d'août 2013 la réforme du régime des injonctions de soins. Le comité parlementaire déplore, entre autres, que l'utilisation de la détention en hôpital psychiatrique, possibilité corrélée à l'injonction de soins, soit utilisée par les psychiatres anglais pour pouvoir trouver des lits à certains patients - la carence de lits est due au manque d'investissement chronique dans les structures hospitalières. Ainsi, la dangerosité criminologique, les risques posés par le patient, sont fréquemment invoqués à mauvais escient, pour contourner les politiques d'austérité mises en place par le gouvernement britannique, avec pour effet pervers obligé d'accroître artificiellement les statistiques sur le lien «trouble mental »/dangerosité. Les technologies de dépistage sont instrumentalisées par les professions psy pour pouvoir mener à bien leur mission thérapeutique à l'égard de personnes en détresse psychique - tant il est vrai que la dangerosité semble être le seul poste encore à même de mobiliser la dépense publique sans susciter trop de controverses.

En France les récents travaux sur la récidive et la dangerosité présentés à diverses autorités françaises tendent à la sobriété. Ainsi le rapport très posé sur la récidive, inspiré par un réformisme calculé pour mettre fin à la dérive sécuritaire. Ce rapport est le fruit de la conférence de consensus, mécanisme inédit, réunie à l'initiative de Mme Taubira, la Garde des Sceaux. Cette conférence était présidée par Françoise Tulkens, professeur à l'École de Criminologie de Louvain-la-Neuve puis juge et Vice-présidente de la Cour Européenne des Droits de l'Homme. Dans son rapport, « le jury souhaite l'abandon de tous les mécanismes automatiques d'aggravation des peines ou de limitation des possibilités de leur aménagement, y compris pour les condamnés en état de récidive. [...] S'agissant des mesures de sûreté, le jury considère qu'elles sont particulièrement attentatoires aux libertés individuelles et qu'à ce titre elles ne peuvent puiser leur justification dans une notion aussi floue que celle de dangerosité. En conséquence, il recommande d'abolir la rétention de sûreté et la surveillance de sûreté 51 ».

49. Burns et al. "Community treatment orders for patients with psychosis (OCTET): a randomised controlled trial”, The Lancet, vol. 381, 2013, pp. 1627-1633.

50. Voir le rapport du House of Commons Health Committee Review of the 2007 Mental Health Act, http://www.publications.parliament.uk/pa/cm201314/cmselect/cmhealth/584/ 58402.htm (consulté le 28 août 2013). 
Le jury de la conférence préconise aussi l'abandon des peines planchers pour les récidivistes ainsi que la fin des peines automatiques, pour que soit possible un vrai retour à l'individualisation des peines. Selon les nombreux spécialistes entendus par la conférence, il faut que la sentence serve non pas tant à punir qu'à (ré)insérer, car c'est là le seul vrai dispositif de prévention. La loi no 2014-896 du 15 août 2014 relative à l'individualisation des peines et renforçant l'efficacité des sanctions pénales tente de respecter les grandes orientations du rapport en dépit des habituelles accusations de laxisme de l'opposition ${ }^{52}$. La loi adopte la recommandation de suppression des peines planchers, supprimées à compter du 1 ${ }^{\text {er }}$ octobre 2014, et se recentre sur une individualisation des peines visant à prévenir la récidive au cas par cas plutôt qu'en soutenant une politique indiscriminée d'incapacitation maximale de tout individu présumé dangereux. Reste à voir comment la nouvelle peine de probation, rebaptisée contrainte pénale et censée s'appliquer aux peines encourues de cinq ans et moins ${ }^{53}$, sera appliquée par le judiciaire une fois la loi en vigueur (il n'y a pas d'automaticité de la contrainte pénale), et si les infrastructures et le financement nécessaires à la réinsertion sociales seront à la hauteur de l'idéal défendu par la ministre. Enfin, la contrainte pénale, même si elle vise à freiner le recours à l'incarcération, s'inscrit malgré tout dans la logique du continuum peine-sûreté déjà mentionné.

Ce rapport préconise également que les expertises médico-psychologiques et psychiatriques doivent rester « centrées sur la responsabilité pénale du condamné et sur l'évaluation des processus psychopathologiques en jeu dans le fonctionnement et l'accessibilité aux soins ». C'est aux professionnels du milieu judiciaire d'évaluer la dangerosité criminologique et le potentiel de récidive ; il ne faut pas mélanger dangerosité psychiatrique et dangerosité criminologique ${ }^{54}$.

51. Conférence de Consensus pour une nouvelle politique publique de prévention de la récidive, op. cit. p. 3.

52. «Taubira joue son va-tout sur la réforme pénale », Le Monde du 3 juin 2014. Voir aussi le discours de Christiane Taubira à l'Assemblée Nationale le 3 juin 2014, disponible sur le site de la Chancellerie, http://www.justice.gouv.fr/la-garde-des-sceaux-10016/pl-prevention-de-larecidive-et-individualisation-des-peines-27180.html (consulté le 5 juin 2014).

53. Et non pas aux condamnations effectives de cinq ans. Ce sont donc les petits délits qui sont concernés par cette nouvelle peine. "Selon la chancellerie, la contrainte pénale ne devrait être appliquée qu’à 8000 à 20000 des 600000 condamnations correctionnelles annuelles. » Ibid.

54. «Lors de l'audition publique organisée par la Fédération française de psychiatrie sur l'expertise psychiatrique pénale en janvier 2007, les recommandations sur l'évaluation de la dangerosité dans l'expertise ont défini la dangerosité psychiatrique comme une "manifestation symptomatique liée à l'expression directe de la maladie mentale" et la dangerosité criminologique comme "prenant en compte l'ensemble des facteurs environnementaux et situationnels susceptibles de favoriser l'émergence du passage à l'acte" [...] Ces définitions de la dangerosité ne sont pas des définitions juridiques. " (HAS 2011, op. cit., pp. 2-4) Par ailleurs, le Rapport Santé-Justice définit la dangerosité psychiatrique comme un risque « de passage à l'acte principalement lié à un trouble mental, et notamment au mécanisme et à la thématique de l'activité délirante. », Rapport Santé-Justice, op. cit., p. 10. 
Les travaux présentés récemment lors d'une audition publique devant la Haute Autorité de Santé française sont également mesurés. Ce rapport de 2011, qui consiste en une analyse de la littérature et des recherches dans le champ de la dangerosité psychiatrique, déplore que « depuis 1994, le lien entre les questions de réitération, récidive, dangerosité, peine et mesure de sûreté [ait] été constant 55 », et que le soin lui-même en soit venu à être utilisé comme une mesure de sûreté. Le rapport constate aussi que « la peur du crime immotivé, alimentée par les faits divers médiatisés, plaide de façon récurrente en faveur d'un nouveau traitement du danger potentiel représenté par les personnes souffrant de troubles mentaux. Les préoccupations de défense sociale sont ainsi réactivées et les psychiatres sont de nouveau invités à s'engager sur la voie d'un contrôle de populations désignées «à risque ». Le risque ressenti comme imminent d'un passage à l'acte violent n'est plus toléré par la population $56 »$.

Cette représentation politico-médiatique d'une opinion intolérante 57 cause une «tension critique entre les pratiques sécuritaires et l'humanisme de la clinique ». Pour la désamorcer, le rapport produit une analyse exhaustive de la recherche au niveau international afin de montrer que les « personnes ayant des troubles mentaux représentent de 3 à $5 \%$, voire $10 \%$, des actes de violence en général » (les variations s'expliquent par les différences entre les définitions et échelles utilisées), ainsi qu'une moyenne de 1 homicide sur 2058. De plus, même si ce lien existe, les études réalisées dans toute une série de pays comparables montrent également qu'il n'y a pas de lien de cause à effet entre trouble mental et violence. Le lien, si tant est qu'il y en ait un, est multifactoriel, complexifié par la comorbidité notamment avec l'abus de substances psychoactives, ainsi que la présence d'un environnement violent ou de facteurs d'exclusion sociale - ce qui est le cas d'ailleurs pour la quasi-totalité des comportements délinquants. Toutes les études s'accordent à conclure que pour ce qui est du rapport entre trouble mental et violence, il s'agit de corrélation plutôt que de causalité. Par contre les personnes souffrant de troubles mentaux sont bien plus fréquemment victimes de violences que la population dite «saine », ce qui n'entre jamais en considération dans les débats sur la prévention des risques ${ }^{59}$. Enfin, le rapport est ferme sur ces deux points : identifier la violence à la maladie mentale entraîne beaucoup de «faux positifs 60 »; et surtout, la meilleure stratégie préventive pour les personnes souffrant de troubles mentaux et susceptibles de passages à l'acte violents (les seuls réels facteurs prédictifs étant des antécédents de violence subie ou infligée et non pas le

55. HAS 2011, op. cit., p. 7.

56. Ibid., p. 6.

57. En Angeleterre la British Crime Survey essaye depuis plus de dix ans de produire une représentation de l'opinion publique qui soit distincte de l'interprétation qu'en donnent les discours politiques et les médias.

58. HAS 2011, op. cit., p. 10.

59. Ibid., p. 22.

60. Ibid., p. 33. 
trouble mental) est l'alliance thérapentique réelle et construite sur la durée entre le patient et l'équipe thérapentique; elle implique une «clinique du quotidien $61 »$. Ainsi le rapport, qui n'est pas un rapport d'opinion mais une analyse de la recherche dans de nombreux pays comparables, est donc très clair : le dépistage de la dangerosité a très peu de potentiel préventif, contrairement au travail clinique soutenu sur le long terme avec des personnes fragiles, dont les éventuels passages à l'acte sont bien plus souvent une réaction à une intrusion intolérable, à une souffrance non entendue, plutôt que le fait d'une pulsion perverse, monstrueuse ou malsaine.

Les psychiatres français, qui réclament l'abrogation de la loi de juillet 2011 sur les soins psychiatriques sans consentement, se servent aussi des voies de recours juridictionnelles afin de lutter contre les demandes sécuritaires dont fait l'objet leur profession : l'association Cercle de réflexion et de proposition d'actions sur la psychiatrie (CRPA) a récemment soulevé une question prioritaire de constitutionnalité concernant la loi de 2011 sur les soins psychiatriques sans consentement et a obtenu l'abrogation de deux dispositions 62 concernant le traitement de personnes souffrant de troubles mentaux et ayant commis des infractions pénales ${ }^{63}$. Suite à cette décision et avant de légiférer à nouveau, le gouvernement a demandé un nouveau rapport. La même association a aussi saisi la Cour européenne des Droits de l'Homme le 12 mai 2014 de la validité du régime des soins psychiatriques sans consentement en France.

Il existe donc des stratégies réformistes efficaces, qui font frein à un utilitarisme dur face aux singularités en jeu. Cependant le réformisme ne sort pas le débat public de son enlisement dans un va-et-vient constant entre les tenants des droits de l'homme et les inconditionnels de la sécurité, où le seul enjeu est de savoir où placer le curseur sur le continuum liberté-sécurité. La clé de voute conceptuelle, la notion de dangerosité, est intouchée.

\section{Politique de la vérité : problématiser la dangerosité}

\section{Quel rôle pour l'intellectuel?}

Foucault s'est beaucoup intéressé aux effets de vérité qu'il est possible, souhaitable, de produire à partir d'une position d'énonciation : celle de l'intellectuel, puis du philosophe ${ }^{64}$. L'intellectuel, le philosophe, n'ont pas à proposer un savoir alternatif au savoir hégémonique, mais à intervenir de façon stra-

61. Ibid., p. 48.

62. Au motif que les garanties légales pour la mainlevée des personnes détenues en UMD (Unité malades difficiles) n'étaient pas suffisantes, ainsi que de l'insuffisance de l'information des personnes détenues suite à une infraction.

63. Décision n 2012-235 QPC, Conseil constitutionnel, 20 avril 2012, qui a donné lieu à la «Proposition de loi visant à modifier certaines dispositions issues de la loi n ${ }^{\circ} 2011-803$ du 5 juillet 2011 relative aux droits et à la protection des personnes faisant l'objet de soins psychiatriques». 
tégique sur certains points bien choisis. Ainsi par exemple dans «Enfermement, psychiatrie, prison », il affirme : "Ma position, c'est qu'on n’a pas à proposer. Du moment qu'on “propose”, on propose un vocabulaire, une idéologie, qui ne peuvent avoir que des effets de domination. Ce qu'il faut présenter, ce sont des instruments et des outils que l'on juge pouvoir être utiles ainsi. En constituant des groupes pour essayer précisément de faire ces analyses, de mener ces luttes, en utilisant ces instruments ou d'autres, c'est ainsi, finalement, que des possibilités s'ouvrent 65 ». Et quand, dans la dernière période de son enseignement, Foucault traite des pratiques de soi, des lignes de subjectivation et de l'éthique, le philosophe est aussi celui qui fait appel aux « capacités éthiques 66 » des acteurs d'un dispositif donné.

Dans cette optique de résistance aux effets de domination du langage en tant que tel, le réformisme pragmatique ne suffit pas. Il ne faut ni consentir aux termes du débat en présence, ni céder sur une critique radicale des institutions pénales, de leur fonctionnement, de leurs objectivités, ou encore des formes de subjectivités qu'elles produisent. Sur le versant « politique de la vérité » donc, il s'agira cette fois de problématiser la notion de dangerosité elle-même et non plus seulement de tempérer les excès de son application dans la pratique.

Problématiser, pour Foucault, c'est faire « l'histoire du présent 67 »: démontrer la contingence des discours à valeur de vérité, exposer les aléas de leur mise en place, restituer leur statut d'événement à certains concepts ou certaines techniques qui aujourd'hui apparaissent incontournables, mettre à jour les enjeux de pouvoir, rendre visibles les régularités discursives d'un même épistème - la liste n'est pas exhaustive ; les tactiques et stratégies inventées par Foucault varient selon les champs, les discours, les pratiques, les buts stratégiques.

Foucault va s'intéresser au terme de dangerosité à plusieurs reprises : en effet tout un pan de sa réflexion vise la «manière dont [les sociétés] se débarrassent non pas de leurs morts, mais de leurs vivants... 68 ». Et la dangerosité

64. Voir notamment sur la problématisation de la position d'énonciation du philosophe les deux derniers cours au Collège de France sur la parrêsia, Le Gouvernement de soi et des autres, 1982-3, Paris, Gallimard/Le Seuil, 2008 et Le courage de la vérité. Le gouvernement de soi et des autres II. Cours au Collège de France 1984, Gallimard/Le Seuil, 2009. Voir aussi la situation du cours de Fabienne Brion et Bernard Harcourt, Mal faire, dire vrai : fonctions de l'aveu en justice, Louvain-la-Neuve, Presses Universitaires de Louvain, 2012.

65. Foucault M., in «Enfermement, psychiatrie, prison », op. cit., p. 348.

66. Sur la problématisation comme stratégie, voir la situation de cours de Fréderic Gros, in Michel Foucault, Le Gouvernement de soi et des autres 1982-3, op. cit. Par exemple : "Cette nouvelle manière de faire de la politique en procédant par problématisation plutôt que par dogmes, en misant sur les capacités éthiques des individus plutôt que sur leur adhésion aveugle à des doctrines », p. 360.

67. Voir aussi Castel : «C'est le recours à l'histoire qui donne son épaisseur au présent et permet d'y dégager des enjeux dépassant la simple phénoménologie descriptive des pratiques », Castel R., La gestion des risques, op. cit., p. 200. 
est une notion opératoire pour l'exclusion des vivants indésirables, de ces « résidus 69 » que sont le délinquant et le fou, de par la double lecture juridique et psychologique qu'elle permet. Foucault s'est donc attaché à problématiser cette notion ségrégative en interrogeant ses conditions de possibilité ainsi que la manière dont elle en était venue à acquérir un statut d'objectivité dans la pénologie moderne. Pour faire la généalogie de ce concept, il convoque la psychiatrie - en tant que pouvoir -, l'expertise psychiatrique - en tant que technologie -, et la subjectivité criminelle - en tant que mode d'assujettissement ${ }^{70}$.

\section{Rapports droit-psychiatrie : la mainmise du pouvoir psychiatrique}

Aujourd'hui, que les psychiatres aient un rôle à tenir dans le système pénal ne fait pas question. Or, dans son avant-propos à Crime et folie : deux siècles d'enquêtes médicales et judiciaires, Renneville, historien du droit aujourd'hui employé par l'Administration pénitentiaire, nous rappelle que jusqu'à la fin du XVIII ${ }^{\mathrm{e}}$ siècle, " le droit considère que le criminel est maître de sa raison et que le fou ne saurait être criminel $71 »$. Droit et discipline asilaire pouvaient alors être représentés comme deux ensembles à intersection vide : le criminel relève soit de la folie, soit de la justice. Or cette séparation s'efface graduellement et l'on passe d'un régime dans lequel la folie criminelle n'est pas punie par la justice, car elle est le signe d'une déraison totale, à une multiplicité de figures de la déraison partielle et donc relevant du système pénal. On voit ainsi apparaître les termes de monstres, pervers, anormaux ; toutes les formes de la délinquance deviennent susceptibles d'appeler le soupçon de déraison. Aujourd'hui encore, « toujours sollicitée pour combler les fractures du lien social, la psychiatrie poursuit sa quête de l'anormalité criminogène avec l'appui de nouvelles sciences, comme la génétique ou la neurobiologie $72 »$.

68. Foucault M. in « Normalisation et contrôle social », op. cit., p. 319.

69. Le mot est de Foucault. On le trouve notamment dans Le pouvoir psychiatrique. Cours an Collège de France. 1973-1974, Paris, Gallimard/Le Seuil, 2003.

70. Outre le texte sur « l'individu dangereux », les textes-clés de cette généalogie sont le livre édité par Michel Foucault à partir du travail en séminaire (conduit en parallèle à son Cours au Collège de France) sur Pierre Rivière, la leçon 10 du séminaire de 1973-4 intitulé Le pouvoir psychiatrique et contemporain au dit séminaire et la leçon 6 du Cours à Louvain de 1981, Mal faire, dire vrai. Il faut aussi garder à l'esprit que le cours sur le pouvoir psychiatrique est suivi du cours sur Les anormanx. Cours an Collège de France. 1974-1975, Paris, Gallimard/Le Seuil, 1999, et celui de 1975-1976, Il faut défendre la société. Cours an Collège de France. 1975-1976, Paris, Gallimard/Le Seuil, 1997, contemporain avec Surveiller et punir. Le cours de 75-76 n'est pas tellement un changement de sujet pour Foucault mais une ouverture plus large sur la défense sociale, dont la psychiatrie n'est qu'un dispositif. On passe donc, entre 75 et 76, de l'hypothèse selon laquelle la psychiatrie est avant tout une discipline destinée à assurer la défense sociale à une situation de la psychiatrie «dans une perspective plus globale sur les moyens qu’à une société de se défendre », Le Blanc G. « Les indisciplinés ou une archéologie de la défense sociale », in Le Blanc, G. et Terrel, J. (eds.), Foucault au Collège de France : un itinéraire, Bordeaux, Presses Universitaires de Bordeaux, 2003, p. 27. De même, Les anormanx ajoute ceci au pouvoir psychiatrique d'inscrire ce pouvoir dans un contexte d'émergence d'un pouvoir de normalisation, p. 24.

71. Renneville M., op. cit., p. 10. 
C'est à la constitution de ce rapport historiquement inédit entre médecine et droit, ainsi qu'à la création d'une série de concepts qui ne sont ni juridiques ni médicaux ${ }^{73}$ par le biais des expertises médico-légales, que Foucault s’intéresse à partir de son cours au Collège de France sur Le pouvoir psychiatrique. En effet, il note, à partir des années 1820-25, ce curieux phénomène : les médecins donnent leur opinion à propos d'un crime, sans sollicitation de l'appareil judiciaire, et essayent de « revendiquer pour la maladie mentale le crime luimême. Devant tout crime, les psychiatres posaient la question : mais est-ce que ce ne serait pas un signe de maladie ? ${ }^{74}$ ». C'est le moment de la naissance de la notion de monomanie homicide, une forme de folie dont le seul symptôme serait le crime. Pourquoi revendiquer «l'appartenance éventuelle du crime à la maladie mentale ? » "Il s'agit, non pas tellement de démontrer que tout criminel est un fou possible, mais de démontrer, - ce qui était beaucoup plus important pour le pouvoir psychiatrique -, que tout fou est un criminel possible. Et la détermination, l'épinglage d'une folie sur un crime, à la limite de la folie sur tout crime, était le moyen de fonder le pouvoir psychiatrique, non pas en termes de vérité, puisque précisément ce n’est pas de vérité qu'il s'agit, mais en termes de danger ; nous sommes là pour protéger la société, puisque, au cœur de toute folie, il y a inscrite la possibilité d'un crime ${ }^{75}$ ». La thèse de Foucault dans le Pouvoir psychiatrique est donc la suivante : ce sont les psychiatres qui se sont positionnés en agents irremplaçables de la défense sociale, qui ont misé sur le contrôle social à défaut de pouvoir fonder leur pratique en vérité. En contrepartie, le pouvoir psychiatrique, en tant qu'il n'est pas confiné à l'espace asilaire, hospitalier, ou institutionnel, mais qu'il nomme un mode de pouvoir 76 inédit dont la spécificité est de faire autorité sur une série de comportements progressivement définis comme pathologiques, a pu se généraliser, au prix néanmoins d’une « impasse sur la thérapeutique 77 ».

72. Ibid., p. 14-15. Voir sur ce point le travail de Nikolas Rose : «The Biology of Culpability: Pathological Identity and Crime Control in a Biological Culture ", Theoretical Criminology, 4-1, 2000, pp. 5-34; " "Screen and Intervene": Governing Risky Brains ", History of the Human Sciences, 23-1, 2010, pp. 79-105.

73. Ainsi la notion de responsabilité atténuée, qui n’a « aucun sens juridique et aucun sens médical », donne lieu à celles d' ' accessibilité à la sanction, la curabilité ou l'adaptabilité. Ces trois notions ne sont ni des notions psychiatriques ni des notions juridiques, mais cela a des effets pénaux qui sont énormes. », Foucault M., "Table Ronde sur l'expertise psychiatrique », Dits et écrits t. 2, op. cit., p. 668.

74. Op. cit., p. 249. Et dans la «Table ronde sur l'expertise psychiatrique»: « Ce qui est frappant dans l'histoire de l'expertise psychiatrique en matière pénale, c'est le fait que ce sont les psychiatres qui, vers 1830, se sont absolument imposés à la pratique pénale qui n’en avait que faire et qui a tout fait pour les écarter. Ils se sont imposés à elle et maintenant ils l'ont en main. » (Foucault M., Dits et écrits t. 2, op. cit., p. 664)

75. Le ponvoir psychiatrique, op. cit., pp. 249-250.

76. Voir aussi dans le texte de 1977 sur « l'individu dangereux »: « si le crime est devenu alors pour les psychiatres un enjeu important, c'est qu'il s'agissait moins d'un domaine de connaissance à conquérir que d'une modalité de pouvoir à garantir et à justifier. ", op. cit., p. 449. Foucault définit cette modalité comme une forme d'hygiène publique.

77. Foucault cité par Le Blanc, op. cit., p. 36. 
Quelques années plus tard, en 1977, Foucault tente une nouvelle problématisation de la dangerosité dans "L'évolution de la notion d'“individu dangereux" dans la psychiatrie légale du XIXe siècle 78 ». Le texte commence par ajouter une dimension biopolitique à l'argument du Pouvoir psychiatrique, cours qui s'inscrivait dans l'analyse du pouvoir disciplinaire. Outre sa fonction de correction des individus, la psychiatrie trouve aussi sa place dans l'arsenal des techniques biopolitiques pour la gestion des populations. L'explosion démographique et la révolution industrielle au XVIIIe siècle ont fait apparaître le corps social, non plus comme « une simple métaphore juridico-politique » mais «comme une réalité biologique et un domaine d'intervention médicale. Le médecin doit donc être le technicien de ce corps social, et la médecine, une hygiène publique 79 ». La psychiatrie s'inscrit dans cette logique de médecine du corps collectif, d'abord par dépistage des «dangers individuels 80 », puis par définition catégorielle des individus dangereux effectuée à partir de ce premier dépistage ${ }^{81}$.

Ensuite, alors que dans Le pouvoir psychiatrique Foucault avait mis l'accent sur les enjeux professionnels de la psychiatrie dans le mouvement de psychiatrisation du droit, dans ce texte il interroge l'acceptation de cette intervention par le milieu judiciaire. La justice, dit-il, trouve intérêt à la mise en intelligibilité de la subjectivité criminelle par la psychiatrie à partir du moment où la sanction est conçue sur le modèle non plus de la punition mais de la correction, comme une «technique de transformation individuelle 82 ». En effet, pour « corriger » efficacement l'individu, il faut un savoir sur sa subjectivité, produit soit lors de l'aveu fait par le délinquant quant à ses motifs ou, lorsque le crime est immotivé ou sans aveu, par ces «spécialistes du motif 83 » que sont désormais les psychiatres, et qui viennent suppléer au silence du criminel. On voit ici se profiler la question du soin, aujourd'hui au cœur de nos pratiques.

Enfin dans ce texte, Foucault fait de l'apparition de la notion de responsabilité pour risque en droit civil une condition de possibilité de la criminalisation de la dangerosité car elle permet au droit pénal de ne pas s'engluer dans la problématique déterminisme/libre-arbitre. Cette nouvelle condition de possi-

78. Op. cit.

79. «L'évolution de la notion d'“individu dangereux" dans la psychiatrie légale du XIX ${ }^{\mathrm{e}}$ siècle », op. cit., p. 450.

80. «Table ronde sur l'expertise psychiatrique », op. cit., p. 665

81. Il y a aujourd'hui par exemple une tendance à assimiler schizophrénie et dangerosité, comme le montre le titre du rapport de 2011 devant la HAS : "Dangerosité psychiatrique : étude et évaluation des facteurs de risque de violence hétéro-agressive chez les personnes ayant des troubles schizophréniques ou des troubles de l'humeur. » Voir aussi l'article bien connu de Feeley M. et Simon J. sur le passage de l'individu au groupe comme objet de la criminologie : Feeley M. et Simon J, “The new Penology : Notes on the emerging Strategy of Corrections and its Implications”, Criminology, 30 (4), 1992, pp. 449-74.

82. "L'évolution de la notion ... ", op. cit., p. 454.

83. Ibid., p. 453. 
bilité représente un apport supplémentaire par rapport au Pouvoir psychiatrique. Les notions de risque et de probabilité causale, à savoir la responsabilité pour risque et non plus pour faute, qui apparaissent en droit civil avec l'industrialisation et l'augmentation des accidents du travail, fournissent l'outil conceptuel qui faisait défaut pour pouvoir rendre un individu responsable non seulement de son acte mais aussi de son être. Ainsi le concept de responsabilité pour risque tente de refermer la béance ouverte dans le droit pénal par la pathologisation du crime et permet à la psychiatrie de faire place à « l'imputabilité sans liberté 84 ».

\section{Autovéridiction, hétérovéridiction et subjectivité criminelle}

On trouve le dernier volet de sa problématisation de la dangerosité dans la sixième leçon, datée du 20 mai 1981, de Mal faire, dire vrai. Foucault y choisit de mettre l'accent sur l'aveu, ou autovéridiction, comme technologie constitutive de la subjectivité criminelle. La pratique généralisée - par contamination par la pratique pénitentielle et mutation des institutions judiciaires - de l'aveu a introduit une dépendance de la justice par rapport à l'autovéridiction du criminel : puisqu'on ne punit plus le crime mais le criminel, encore faut-il savoir qui il est. Dans les cas de crimes sans aveu, on substitue à l'autovéridiction l'hétérovéridiction de l'examen psychiatrique ou psychologique du criminel pour « essayer de faire émerger cette vérité du criminel que le criminel luimême n'est pas capable de formuler 85 ». Ce texte reprend donc l'argument du texte de 1977 quant à la brèche introduite dans le droit pénal par « le sujet avouant » : cependant, alors que ce texte était centré sur la convergence de la biopolitique et du pouvoir disciplinaire dans la gestion de la dangerosité (l'aveu n'y figurant que comme point d'ancrage de la psychiatrie qui propose de juger le criminel sur ce qu'il est et non à partir de sa responsabilité), le séminaire de Louvain repositionne les choses dans la perspective de la subjectivité.

Les techniques d'hétérovéridiction font apparaître ce qui était vraiment recherché dans l'aveu : « on ne cherchait pas simplement à faire apparaître le sujet de droit auquel on demande compte d'un délit commis, mais on cherche aussi à faire émerger une subjectivité qui entretient à son crime une relation signifiante. C'est à partir de là, je crois, que commence la question de la connaissance du sujet comme sujet criminel $86 »$.

Foucault rapporte ce dédoublement de l'aveu - entre un dire vrai sur les faits et une ouverture sur les questions de la subjectivité ou un dire vrai sur la subjectivité - à une dernière condition de possibilité historique, rajoutant ainsi un élément à sa problématisation de la dangerosité : outre l'apparition de la responsabilité pour risque en droit civil, une autre transformation se produit

84. Ibid., p. 462.

85. Op. cit., p. 211

86. Idem. 
au même moment, elle aussi ailleurs que dans le droit pénal. Il vise ici la constitution de l'herméneutique du sujet à la fin du XIX ${ }^{\mathrm{e}}$ siècle, qui implique, dans la pratique de la spiritualité chrétienne, une mise au jour des secrets de la conscience par examen de soi et verbalisation à autrui. Avec Freud et la psychanalyse, l'herméneutique s'ouvre sur une pratique du déchiffrement du sujet comme s'il était un texte : pour « enraciner les comportements d'un sujet dans un ensemble significatif 87 ». Dès lors, le crime se constitue en acte significatif, et sa signification se substitue à la notion de causalité dans la pratique pénale. Foucault introduit donc un nouveau déplacement : ce qui est demandé dans l'aveu ce n'est plus la reconnaissance du crime afin de fonder la sentence mais bien l'aveu de « qui tu es », au niveau « de ce qu'est l'être même de la subjectivité criminelle 88 ». Ce déplacement radicalise la béance déjà identifiée en 1977 pour ce qui est du concept de responsabilité pénale, et aujourd'hui en effet seule l'absence de discernement motive l'irresponsabilité ; pour le reste, le criminel est responsable de son être, dont le crime fait signe.

Ce dernier axe de réflexion foucaldien vient rendre compte de l'évolution des pratiques judiciaires en matière de peine, à savoir qu'aujourd'hui la tendance n'est plus à considérer les difficultés psychologiques de l'infracteur comme des circonstances atténuantes mais plutôt comme un facteur d'aggravation de la peine (prolongement en peine de sûreté, obligations de soins, conditions onéreuses du suivi socio-judiciaire). En effet, si le crime n'est que le signe de l'être du sujet, il est donc légitime de déchiffrer, aujourd'hui le comportement du sujet au moyen des savoirs psychiatriques, psychologiques ou criminologiques, demain son matériel organique même (imagerie cérébrale, gènes, hormones). Ces pratiques d'hétérovéridiction fonctionnent sans faire appel à la parole de l'infracteur, sans miser sur ses capacités de subjectivation des dispositifs dont il est l'effet. Elles ne peuvent que mener au pire, car les sujets qui tombent sous le coup de la loi pénale sont de ce fait assujettis à des discours objectivants et soumis au regard scrutateur des évaluateurs de comportement sans que leur soit apporté un soutien réfléchi, orienté par une éthique, leur permettant de trouver un point d'appui, une distance vis-à-vis de leur acte. Cette distance qui, pour tout psychiatre digne de ce nom, est la seule vraie possibilité de prévention de la récidive.

\section{Le danger n'est pas là où on le croit}

Cette généalogie plurielle de la notion de dangerosité telle qu'elle opère aujourd'hui comme outil ségrégatif des vivants indésirables ainsi que comme dispositif constitutif des subjectivités dites criminelles ${ }^{89}$ nous permet d'avoir un regard lucide sur ce qui est aujourd'hui attendu de la psychiatrie en matière

87. Ibid., p. 224.

88. Ibid., p. 227.

89. Ainsi que le note Denis Salas, une justice utilitariste, qui prétend «punir pour prévenir », n'est en fait qu'un « accélérateur de criminalité », La justice dévoyée, op. cit., p. 75. 
pénale. Cependant les concepts de gestion des risques, de précaution, de prévention, sont devenus les nouvelles objectivités qui organisent aussi bien le discours pénal que ses critiques. Ils ne nous permettent plus de saisir les sombres enjeux qui se jouent en-deçà de la rationalité. Que le discours soit politique ou critique, il offre peu de prise au psychiatre tenté de développer des pratiques cliniques et médico-judiciaires qui à la fois fassent sens théoriquement et soient éthiquement défendables 90 . En effet, comment un psychiatre, un acteur de la justice, peut-il résister à la demande d'orienter son action sur la défense sociale ? Ce n'est certes pas en dénonçant le caractère actuariel ou managérial des pratiques pénales, car après tout, la demande qui lui est adressée n'est-elle pas raisonnable ? Il ne peut que s'agir pour chaque sujet - psychiatre, expert, juge, travailleur social, psychologue en milieu carcéral, etc. - d'une prise de parti éthique : celle de faire exister la singularité dont il fait le pari qu'elle subsiste au-delà de la catégorie « à risque » chez chaque individu, quels que soient par ailleurs les degrés ou nature de sa "folie ». Mais à quel titre, cette prise de parti ?

Il faudrait relire, encore et toujours, La naissance de la clinique : une archéologie du regard médical. Ce beau livre de Foucault, qui explore les conditions de possibilité de la naissance de la médecine, montre aussi la centralité de la volonté de savoir et de voir qui anime le discours médical, ainsi que la réduction du corps individué à un objet pour le regard ${ }^{91}$. À la lumière de cet ouvrage, il est clair que ce qui est dangereux, c'est avant tout de faire l'économie d'une problématisation toujours à renouveler des pulsions et volontés de jouissance qui trouvent à se loger sous le nom d'objectivité scientifique. Ce danger, Foucault le situe aussi au cœur même des pratiques judiciaires à propos de la fonction dés-angoissante de l'expert : « je crains qu'il ne soit dangereux de laisser les juges continuer à juger seuls en les libérant de leur angoisse et en leur évitant de se demander au nom de quoi ils jugent, et de quel droit, qui, quels actes, et qui ils sont, eux qui jugent. Qu'ils s'inquiètent comme nous nous inquiétons d'en rencontrer parfois de si peu inquiets ! 92 ». Ces quelques lignes sans compromis remettent le danger à sa place : en effet, ce que Foucault s'est évertué à démontrer au cours de son enseignement, ce n'est pas tant le fonctionnement liberticide des mécanismes de pouvoir, mais que ce qui est dangereux, c'est de faire l'économie de sa division subjective dans l'exercice d'un pouvoir sur le vivant.

90. Et ainsi éviter la dérive de la psychiatrie ainsi épinglée par Elisabeth Roudinesco : « une psychiatrie sans clinique ni éthique, au service d'un État anonyme plus barbare que les barbares qu'il prétend désigner. », in Roudinesco E., "L'CEuvre de Foucault à l'épreuve de la nouvelle psychiatrie », in Justice et folie, op. cit., p. 43.

91. Foucault M., La naissance de la clinique : une archéologie du regard médical, Paris, Presses Universitaires de France, 1963. Pour une lecture critique de ce texte, voir Voruz V., "The Status of the Gaze in Surveillance Societies » in Golder B. (ed.), Re-Reading Foucault: On Law, Power and Rights, London \& New York, Routledge, 2012.

92. Foucault M., «L'angoisse de juger » (entretien avec R. Badinter et J. Laplanche) in Foucault M., Dits et écrits t. 3, op. cit., p. 297. 